\title{
Towards a Healthy Society: Identifying Socio-Cultural Factors that Might Influence Disabling Addictive Behaviors in Working Populations
}

\author{
Gabriela Walker ${ }^{1}$, Bernard W. M. Wone ${ }^{2}$ \\ ${ }^{1}$ Division of Curriculum \& Instruction, ${ }^{2}$ Department of Biology \\ University of South Dakota
}

\begin{abstract}
This study explores the possible socio-cultural factors affecting addictive behaviors leading to disability in work-aged populations. A sparse generalized canonical correlation analysis was used to model and correlate socio-cultural factors with high addictive behaviors (drug, tobacco, alcohol, and food over-consumption) in 15 to 49 year olds that might impact work place. The results revealed that both within and between the socio-cultural and the addictive group factors there is a great degree of homogeneity, with less developed countries exhibiting lower addictive behaviors (such as food consumption for males and females, tobacco use for females, drug use for females) as the culture of these countries exhibited less information flow, social globalization, personal contact, cultural proximity, but more gender inequality. These results suggest that the level of development of a country is positively correlated to addictive behaviors, addiction-attributable disability, and early death, despite more personal contact, information flow, and cultural proximity, social globalization, national happiness level, individualism, and higher gross domestic product.
\end{abstract}

\section{Introduction}

One of the main tenets of the World Health Organization (WHO) is everyone's right to the highest possible status of health. Gostin et al. [1] built the argument that if the next WHO Director General will focus on this structural value, "ensuring the universal right to health" by creating a Framework Convention on Global Health, then subsequent healthrelated issues can also find a faster and easier solution. The present study pertains to understanding influences of different factors on Years of Lived with a Disability (YLD) (i.e., years of life when a specific functional impairment is manifested, as caused by a disease) and Disability-Adjusted Life Year (DALY) (i.e., overall disease burden) stemming from behaviors related to alcohol, drug, tobacco, and food consumption in the 15 to 49 age group. Understanding the impact of socio-cultural factors on (the length of years lived with) disabilities is complex, however, looking into how workers deal with stress at the peak of their productive years, may enable researchers to study further both labor stress and different solutions that various cultures employ to release stress.

As such, research of this type is exploratory in nature, and not confirmatory of hypotheses. The overarching umbrella of 'socio-cultural factors' a nation, or people, exhibit cannot be completely captured in standardized indices, and can certainly provide only a static and temporal slice of the state of addiction disability within a nation at the moment in time when the data were collected. However, because (1) global research in different fields is much more advanced today than ever before, allowing for different indices to be computed, weighted, and tested for robustness; (2) because of an increased adherence to international organizations and forums which require datareporting; (3) because of more powerful statistical methodologies and software that can handle multiplelayered global data; (4) because of a raise in transparency of government and international mechanisms; (5) because of an increase advocacy for social justice, equity, etc.; and (6) despite the temporality and partiality in a continuously-moving scene of the data (and, hence, of the results), the study question does not become less interesting or less important, especially that is has not been asked, nor answered, before. For this study, we narrowed down the socio-cultural environment to 17 factors related to societies and individual general well-being. In addition, the Human Development Index (HDI) is used as a categorical mediator to facilitate in data analysis. Also, the economic factors are so diverse within each country, and in most world countries, from privatized firms to governmental work, that we held the economic factors as ceteris paribus factors, considering, however, the Gross Domestic Product to provide a measure of the social theoretical concept: the 'culture of poverty'.

In 2010, Martens, Akin, Maud, and Mohsin [2] inquired whether globalization is healthy, using the Maastricht Globalization Index against the infant, under-five, and adult mortality rates. The study concluded that high globalization correlates with low levels of mortality at all ages. When correcting for confounders (such as, Gross Domestic Product (GDP); water quality; health expenditures and financing; smoking; secondary education; and public health resources), all mortality indicators have raised their explanatory power, meaning that they play a significant role in these relationships. Technological 
and socio-cultural globalizations were especially strongly correlated to the mortality indicators.

A recent global study looking at smoking prevalence and attributable disease burden concluded that smoking was the leading risk factor for cancers and chronic respiratory diseases, remaining among the leading risk factors for early death and disability in more than 100 countries in 2015 [3]. Using the Socio-Demographic Index (SDI) (a new summary measure of overall development from Global Burden of Disease 2015 study, formed of average income per person, educational attainment, and total fertility rate), this study found that population growth and ageing contributed to an increase in smoke prevalence and subsequent smoking-attributable diseases in low to middle SDI geographies.

Leading medical journals have published multiple studies which link the dual burden of over nutrition and undernutrition among disadvantaged populations [4] to illicit drug use [5], and both are significantly correlated to poverty $[6,7,8]$. In terms of alcohol, a recent study reveals that socioeconomic status (SES) is an effect modifier of alcohol consumption and its side effects, with a low SES being correlated to increased alcohol-attributable diseases [9].

\section{Method}

\subsection{Independent variables}

Seventeen explanatory variables were considered for this exploratory study to illustrate the social and cultural contexts in which people aged 15 to 49 live and work under.

(a) KOF Globalization Index (KOF ETH Zurich, 2010), with the following indices and subindices (according to the Definitions and Sources document):

(1) Social Globalization Index ( $s g$ ) as a general measure of the following variables:

(2) Personal Contact Sub-Index ( $p v)$, including telephone traffic; transfers of goods, services, income, or financial items as percent of GDP; international tourism; foreign population; and international letters.

(3) Information Flow Sub-Index (ifs), including internet users, television, and trade in newspapers.

(4) Cultural Proximity Sub-Index ( $c p)$, including number of McDonald's restaurants, Ikea stores, and trade in books.

(b) (5) Adult Literacy Rate (alr), population $15+$, both sexes, extracted from Data World Bank.

(c) Education level [10]:

(6) Average school attendance for females, per country - country school life expectancy (primary to tertiary education years) (CLEF)

(7) Average school attendance for males, per country - country school life expectancy (primary to tertiary education years) (CLEM) (d) (8) National Happiness (Happiness), reflecting a "growing global interest in using happiness and subjective well-being as primary indicators of the quality of human development" [11], coming out of the Sustainable Development Solutions Network (SDSN) and commissioned by UN Secretary-General Ban Ki-moon in 2012, and using a Gallup World Poll methodology.

(e) (9) Religious Diversity (RDI) is calculated based on the shares of eight major world religions (Buddhism, Christianity, folk or traditional religions, Hinduism, Islam, Judaism, other religions considered as a group, and the religiously unaffiliated) [12].

(f) Geert Hofstede dimensions measuring national culture [13], defined by the Hofstede Institute as follows:

(10) Power Distance (PDI), as "the extent to which the less powerful members of institutions and organizations within a country expect and accept that power is distributed unequally."

(11) Individualism (idv), as "the degree of interdependence a society maintains among its members."

(12) Masculinity (mas), as "what motivates people, wanting to be the best (Masculine) or liking what you do (Feminine)."

(13) Uncertainty Avoidance (uai), as "the extent to which the members of a culture feel threatened by ambiguous or unknown situations and have created beliefs and institutions that try to avoid these."

(14) Long Term Orientation (ltowvs), as "how every society has to maintain some links with its own past while dealing with the challenges of the present and future."

(15) Indulgence versus restraint (ivr), as "the extent to which people try to control their desires and impulses."

(g) (16) Gender Inequality Index (GII) measuring the gender equity degree in each country.

(h) (17) Gross Domestic Product (GDP) per capita as a measure of poverty [14].

\subsection{Dependent variables}

The dependent variables on addiction have been extracted from the Global Health Data Exchange [15], and they fare out in DALY (Disability-Adjusted Life Year, a measure of overall disease burden) and YLD measure (Years Lived with the Disability, a measure of the risk behavior of substance consumption for people living with the health condition and its consequences). Four major substances are known to produce addiction: alcohol, drugs, tobacco, and food. Addiction has been linked to depression as a cause, but also to loss of quality of life, short- and long-term disability, and premature death as effects [16]. 


\subsection{Data analysis}

A sparse generalized canonical correlation analysis (SGCCA) was employed to identify and measure the associations between two sets of variables. Classical canonical correlation analysis (CCA) determines the orthogonal linear combination of variables that best explain the variability in both within and between sets and this new combination of variables are called variates or components [17]. Sparse CCA (SCCA) minimizes the number of features used in projections, while maximizing the correlation between the two data sets [18]. Variable selection version of SCCA to three or more sets of variables is SGCCA [19]. More importantly, within the SGCCA framework, the data sets are not necessarily fully connected, which makes SGCCA a flexible method for analyzing a wide variety of practical problems [19]. SGCCA is performed using the $R$ package mixOmics v6.1.3 [20].

\section{Results}

Sparse generalized canonical correlation analysis revealed some interesting patterns associated with social and health factors, and the state of development within the countries. The first variate captured much of the variation in the data sets, with $40 \%$ explained variation for the social variables and $32 \%$ explained variation for the health variables (see Figure 1). The second variate captured $13 \%$ explained variation for the social variables and $9 \%$ explained variation for the health variables. The first social variate selected five social variables (i.e., Information Flow, Social Globalization, Personal Contact, Cultural Proximity, and Gender Inequality), whereas the second social variate selected six social variables (i.e., Power Distance, Religious Diversity, Indulgence versus restraint, National Happiness, Gross Domestic Product, and Individualism) as important social factors in differentiating the developmental status of the countries. The first health variate selected seven health variables (i.e., Male Body Index Years Lived with Disability and Disability-Adjusted Life Year, Female Tobacco Years Lived with Disability and Disability-Adjusted Life Year; Female Mass Body Index Years Lived with Disability, Female and Male Drug Disability-Adjusted Life Year), similarly the second health variate also selected seven health variables (i.e., Male Tobacco Years Lived with Disability and Disability-Adjusted Life Year, Female Mass Body Index Years Lived with Disability and Disability-Adjusted Life Year, Male Mass Body Index Disability-Adjusted Life Year, and Female Alcohol and Tobacco Years Lived with Disability) as important health factors in differentiating the developmental status of the countries. These selected variables are highly correlated (i.e., > 0.5 absolute correlation) with the variates, as well as between variable type (i.e., between the social variables and the health variables; see Figure 2). Overall, additive behaviors, such as tobacco use and Mass Body Index are highly correlated with Information Flow (ifS), Social Globalization (sg), Personal Contact (pv), Cultural Proximity (cp), National Happiness (Happiness), Gross Domestic Product (GDP), and Individualism (idv). Notably, Gender Inequality Index (GII) and Power Distance (PDI) are negatively correlated with the health variables.

\section{Discussion}

In this study, we used a multivariate approach to better understand the relationships between sociocultural factors and addictive behaviors. Our results provide insights into the type of social-cultural factors that might influence addictive behaviors. Specifically, the results suggest that easier access to information and increasing individualism in societies is correlated to rising addiction in that society. In contrast, increasing gender inequality and increased recognition of hierarchical authority (Power Distance) are declining additive behaviors observed in that population.

The findings from the study are intriguing and complex, but, most importantly; it is imperative to state that the correlative results do not necessarily imply causation. With that in mind, the fact that national socio-cultural characteristics influence the length of time that working populations live with disabilities due to addiction together with years lived with disabilities, might have several policy implications. One policy example is Winch and Thomas' [21] approach, illustrating one way of addressing addiction by influencing emotional drivers to promote behavioral changes (such as social inclusion, nurture, disgust) needed in a sufficient quantity and quality so that the individual will react based on the level of sensitivity to the issue. According to the present study, different combinations of these emotional drivers need to be addressed through public education to have an adequate effect in reducing preventing, and treating addiction behaviors. A second policy example in addressing addiction is reevaluating local, national, and international policies. A reanalysis of policies, their implications, and their enforcement might lead to decreased consumption of addictive substances. Although it was shown that increased criminalization of drugs has little effect on reducing drug consumption [22]. For example, a solution at the international level that contributes to the prevention and treatment of addiction is still sought (the Convention on Narcotic Drugs and the Convention on Tobacco Control) are excellent attempts at an international solution, but their effect is trifling. According to the results of this study, a single 
comprehensive solution may be difficult to implement because each country's characteristics make-up is different. However, considering that out of the 11 socio-cultural factors examined, only a handful are common correlates to the four addiction risks; a unifying resolution may be possible with continued research.

These solutions, however, need to be continually evaluated and modified as needed because Berardi (p. 8 [23]) pointed out that "the technical transformation implied in the process of globalization is changing the socio-cultural prospects so deeply that theoretical tools inherited from European critical theory no longer suffice for imagining the future of human evolution."

Lastly, our approach has predictive capabilities. The economic status of two countries (i.e., Iraq and Lebanon) was not designated in the databases, but the model used herein indicates that Iraq is moderately developed, whereas Lebanon is highly to very highly developed. Interestingly, our results suggest that Iraq shows increasing Gender Inequality and Power Distance, whereas Lebanon shows increasing Information Flow, Social Globalization, Personal Contact, and Cultural Proximity. In that respect, Iraq shows increasing male tobacco use, whereas Lebanon shows increasing both male and female Mass Body Index issues.

\section{Conclusion and future research}

In conclusion, the results revealed that both within the socio-cultural and the health/addictive factors there is a great degree of homogeneity, with less developed countries exhibiting lower addictive behaviors (such as food consumption for males and females, tobacco use for females, drug use for females) as the culture of these countries exhibited less information flow, social globalization, personal contact, cultural proximity, but more gender inequality. These results suggest that development of a country leads to addictive behaviors, despite more personal contact, information flow, and cultural proximity. When statistically assessing the effects of socio-cultural globalization, while considering the advanced degree of development of a country, these results are sobering. With increased globalization, development, and equality, we can see an increase in addictive behaviors that lead to disability, loss of quality of life, and, eventually, death (the YLD and DALY measures speak to the burden of disease and lives cut short because of the disability stemming as a result of these addictive behaviors). Public policy, education policy, and health policy lobbyists, lawmakers, governmental institutions, and corporate organizations can use these results to reduce addictive behaviors, decrease disability, decrease dependability of socially-funded disability income, increase the quality of life, and increase the productivity at the work place.

\section{References}

[1] Gostin, L. O., Friedman, E. A., Buss, P., Chowdhury, M., Grover, A., Heywood, M., Kanchanachitra, C., Leung, G., Mackay, J., Matsoso, P., Møgedal, S., Mukherjee, J. S., Omaswa, F., Phumaphi, J., Reddy, K. S., Periago, M. R., Thomas, J., Tomori, O., Were, M., \& Zewdie, D. (2016). The next WHO Director-General's highest priority: A Global Treaty on the Human Right to Health. Lancet, published online October 13, 2016, DOI: http://dx.doi.org/10.1016/S2214-109X(16)30219-4. http://www.thelancet.com/journals/langlo/article/PII S2214-109X(16)30219-4/fulltext?elsca1=etoc

[2] Martens P, Akin SM, Maud H, Mohsin R. (2010). Is globalization healthy: A statistical indicator analysis of the impacts of globalization on health. Globalization and Health (16), Open Access. DOI: 10.1186/1744-8603-6-16.

[3] Gakidou, E., Murray, C. J. L., \& Forouzanfar, M. H. (2017). Smoking prevalence and attributable disease burden in 195 countries and territories, 19902015: A systematic analysis from the Global Burden of Disease Study 2015. Lancet (389), pp. 1885-906. DOI http://dx.doi.org/10.1016/S0140-6736(17)3081 9-X.

[4] Delisle H, Batal M. (2016). The double burden of malnutrition associated with poverty. Lancet (387), p. 2504.

[5] Sigmon, S. C. (2016). Additive burdens of malnutrition, poverty, and substance abuse. Lancet (388), pp. 1879-80. DOI: http://dx.doi.org/10.1016/ S0140-6736(16)31814-1

[6] Davey-Rothwell MA, Flamm LJ, Kassa HT, Latkin CA. (2014). Food insecurity and depressive symptoms: comparison of drug using and nondrugusing women at risk for HIV. Journal Community Psychology (42), pp. 469-78.

[7] Himmelgreen, D.A., Perez-Escamilla, R., SeguraMillan, S., Romero-Daza, N., Tanasescu, M., Singer, M.A. (1998). Comparison of the nutritional status and food security of drug-using and non-drug-using Hispanic women in Hartford, Connecticut. American Journal of Physical Anthropology (107), pp. 351-61.

[8] Mysels, D.J., \& Sullivan, M.A. (2010). The relationship between opioid and sugar intake: review of evidence and clinical applications. Journal of Opioid Management (6), pp. 445-52. 
[9] Katikireddi, S.V., Whitely, E., Lewsey, J., Gray, L., \& Leyland, A.H. (2017). Socioeconomic status as an effect modifier of alcohol consumption and harm: analysis of linked cohort data. Lancet, 2, pp. e267-76. DOI: http://dx.doi.org/10.1016/S2468-2667(17) 30078-6.

[10] CIA. (2017). The World Factbook: School life expectancy (primary to tertiary education). https://www.cia.gov/library/publications/the-worldfactbook/fields/2205.html\#202

[11] SDSN. (2016). The Sustainable Development Solutions Network (SDSN): World Happiness Report. Retrieved from http://worldhappiness.report/

[12] Pew Research Center. (2010). Table: Religious Diversity Index Scores by Country. Retrieved from http://www.pewforum.org/2014/04/04/religiousdiversity-index-scores-by-country/

[13] Itim International. (2016). Geert Hofstede: Country Comparison. Retrieved from http://geerthofstede.com/countries.html

[14] World Data Bank. (2016). World Development Indicators: Gross Domestic Product. Retrieved from http://databank.worldbank.org/data/reports.aspx?Cod e=NY.GDP.PCAP.CD\&id=af3ce82b\&report_name= Popular_indicators\&populartype=series\&ispopular= y\#advancedDownloadOptions

[15] Global Health Data Exchange. (2016). Global Burden of Disease health database. Retrieved from http://ghdx.healthdata.org/global-burden-diseasestudy-2013-gbd-2013-data-downloads-full-results

[16] WebMD. Substance Abuse [Internet]; WebMD, LLC: 2017 [2005-2017]. Available from: http://www.webmd.com/mental-health/addiction/ substance-abuse.

[17] Hotelling, H. (1936). Relations between two sets of variates, Biometrika 28, 321-377.

[18] Hardoon, D.R., \& Shawe-Taylor, J. (2008). Sparse Canonical Correlation Analysis. Centre for Computational Statistics and Machine Learning Department of Computer Science. Published and retrieved from https://arxiv.org/pdf/0908.2724v1.pdf

[19] Tenenhaus, A., Philippe, C., Guillemot, V., Le Cao, K. A., Grill, J., \& Frouin, V. (2014). Variable selection for generalized canonical correlation analysis. Biostatistics 15(3), pp. 569-583.

[20] Le Cao, K.-A., Rohart, F., Gonzalez, I., Dejean, S., with key contributors Gautier, B., Bartolo, F., contributions from Monget, P., Coquery, J., Yao, F.Z., and Liquet, B. (2016). mixOmics: Omics Data Integration Project. $\mathrm{R}$ package version 6.1.1. https://CRAN.R-project.org/package=mixOmics.

[21] Winch, P.J., \& Thomas, E.D. (2016). Harnessing the power of emotional drivers to promote behavior change. Lancet 4(12), pp. e881-2. DOI: http://dx.doi.org/10.1016/S2214-109X(16)30310-2

[22] Room, R., \& Reuter, P. (2012). How well do international drug conventions protect public health? The Lancet (379), pp. 84-91.

[23] Berardi, F. B. (2013). The mind's we: Morphogenesis and chaosmic spasm, pp. 7-32. In Arne De Boever and Warren Neidich (Eds.) "The Psychopathologies of Cognitive Capitalism". Archive Books: Berlin, Germany. 
A.

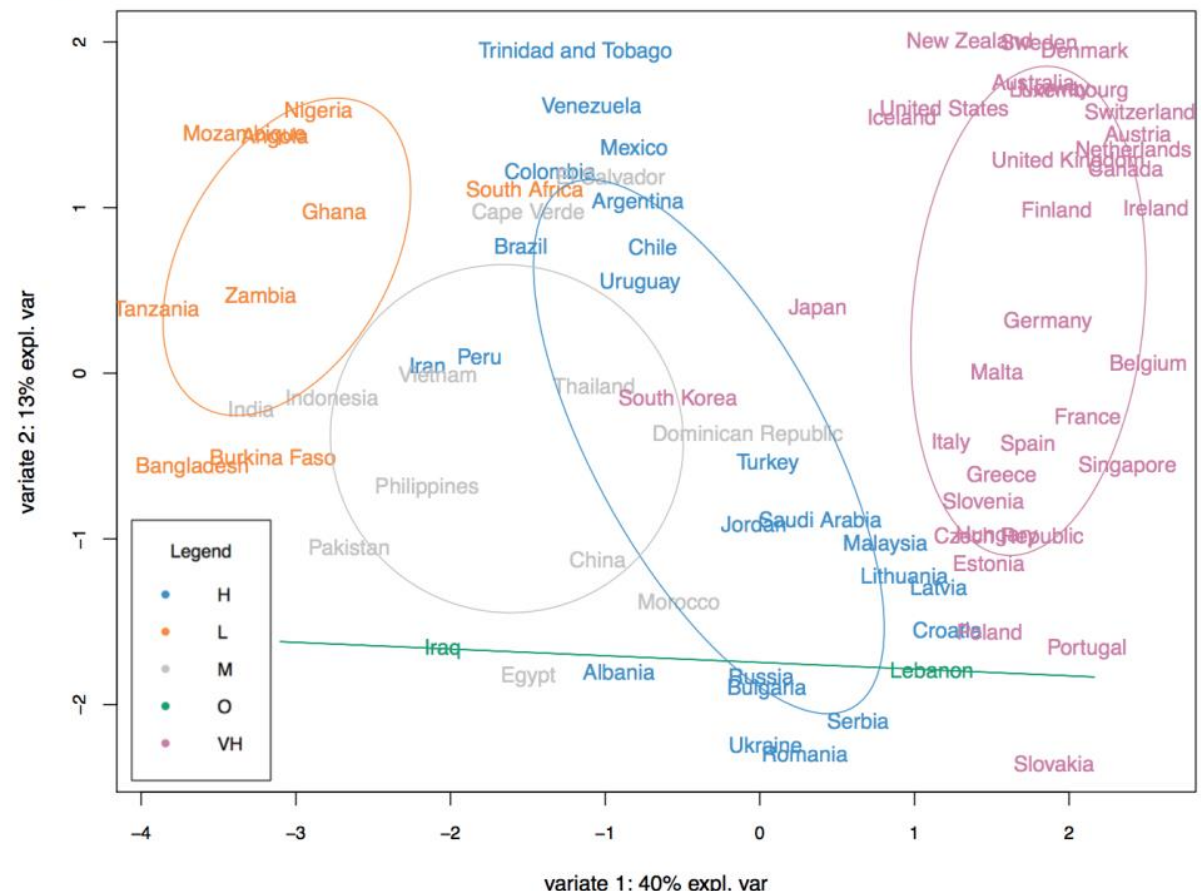

B.

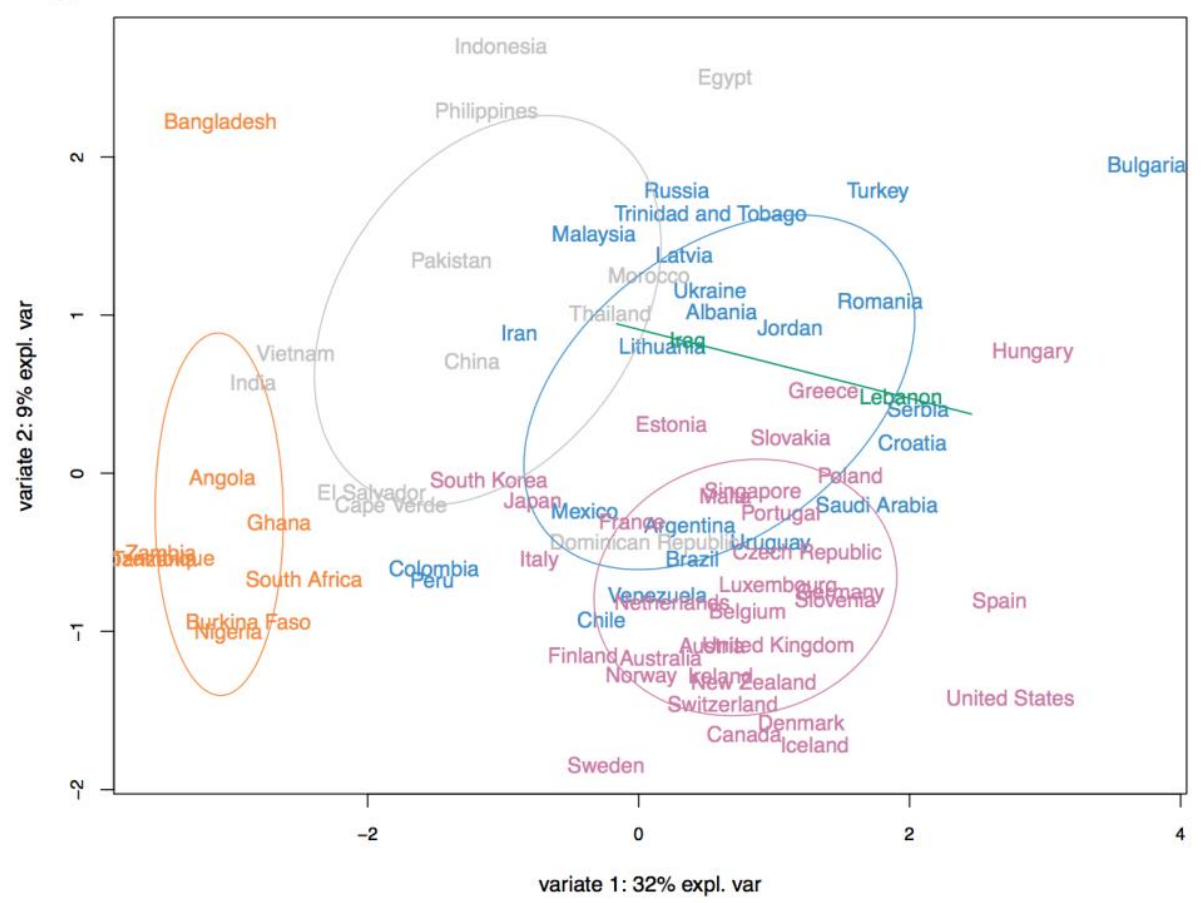

Figure 1. Sparse generalized canonical correlation analysis (SGCCA) reveals interesting patterns in the two data sets (response and explanatory). The first component (i.e., variate 1) differentiated the countries clearly based on their state of development (see legend) whether by (A.) social variables (i.e., explanatory), or by (B.) health variables (i.e., response). Positive variate 1 social scores indicate increasing Information Flow, Social Globalization, Personal Contact, Cultural Proximity, whereas negative variate 1 scores indicate increasing Gender Inequality. Positive variate 2 social scores indicate increasing Indulgence versus restraint, National Happiness, Gross Domestic Product, Religious Diversity and Individualism, whereas negative variate 2 social scores indicate increasing Power Distance. Positive variate 1 health scores indicate increasing additive behaviors (specifically, MBody15YLD, FTobacco15YLD, FTobacco15DALY, MBody15DALY, FBody15YLD, FDrug.15DALY, and MDrug15DALY), whereas negative variate 1 health scores indicate decreasing of the above additive behaviors. Positive variate 2 health scores indicate increasing male tobacco overall disease burden (i.e., MTobacco15DALY, and MTobacco15YLD), and both male and female body image issues (i.e., FBodyIndex15DALY, MBody15DALY, and FBody15YLD), whereas negative variate 2 scores indicate increasing female alcohol and tobacco years lived with the disability (i.e., FAlcohol15YLD, and FTobacco15YLD). Ellipses represent 95\% confidence regions. $\mathrm{H}=$ highly developed, $\mathrm{L}=$ less developed, $\mathrm{M}=$ moderately developed, $\mathrm{O}=$ not designated by databases, $\mathrm{VH}=$ very highly developed. 


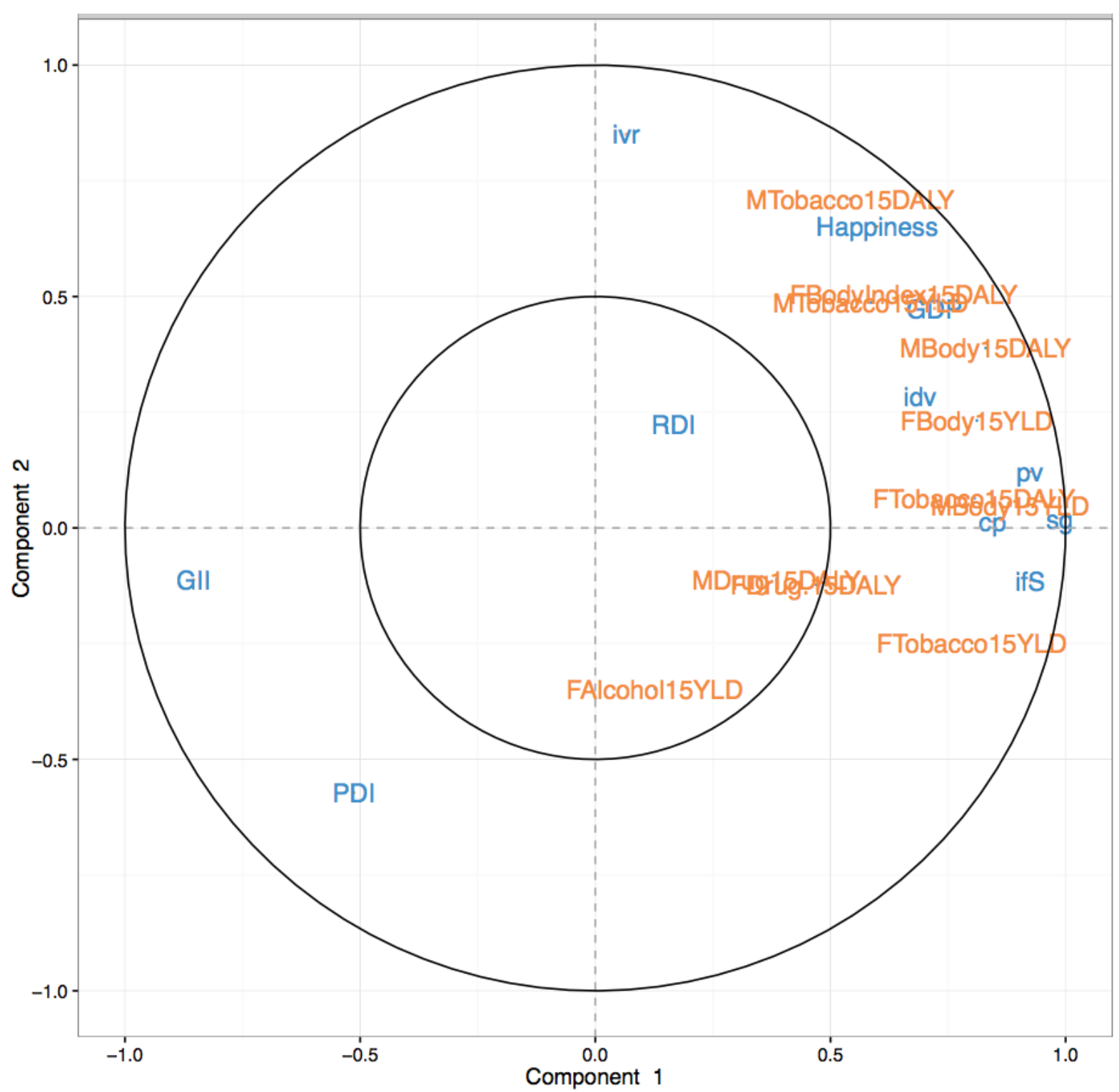

Figure 2. Correlation plot showing which response variables (in orange) are correlated with which explanatory variables (in blue) by looking at formed clusters as defined by the first two components from the sparse generalized canonical correlation analysis. Clustering of variables also indicate their correlations between them. The distance from the origin represents the strength of the correlation (the further from the origin the better). The correlation circle plot also highlights the contribution (absolute scores) of each selected variable to each component (variate). From the biplot, positive Component 1 scores indicate increasing Information Flow (ifS), Social Globalization (sg), Personal Contact (pv), Cultural Proximity (cp), whereas negative Component 1 scores indicate increasing Gender Inequality (GII). Positive Component 2 scores indicate increasing Indulgence versus restraint (ivr), National Happiness (Happiness), Gross Domestic Product (GDP), Religious Diversity (RDI) and Individualism (idv), whereas negative Component 2 scores indicate increasing Power Distance (PDI). Additive behavior variable abbreviations are listed in the paper. 